\title{
GLP-1-Analogon kann das leisten
}

\author{
Die $\mathrm{HbA}_{1 c}$-Reduktion ist nur eine der Aufgaben für ein ideales Antidiabe- \\ tikum. Und das am besten ohne erhöhtes Hypoglykämierisiko. Auch Hy- \\ pertonie, Übergewicht und Dyslipidämie sollte es entgegenwirken, um \\ Komplikationen zu vermeiden. Eine Inkretintherapie könnte das leisten.
}

$(\mathrm{n}=251)$ oder einer 1,8 -mg-Dosis $(\mathrm{n}=247)$ pro Tag erhielten, um $-2,1$ bzw. $-3,6$ mmHg signifikant stärker als bei jenen mit Sulfonylharnstoff $(\mathrm{n}=248)$ mit $-0,7$ $\mathrm{mmHg}(\mathrm{p}<0,05)$. Und die Inkretintherapie wirkt sich offenbar auch auf die Lipide positiv aus: LDL-, Gesamtcholesterin und Triglyzeride nahmen in der LEAD6-Studie $(\mathrm{n}=464)$ mit Liraglutid ab.

Noch ein Pluspunkt: Die Patienten nehmen mit dem GLP-1-Analogon nicht zu wie mit Sulfonylharnstoffen, sondern sogar signifikant ab (LEAD 3). Dabei wird eher das kardiovaskulär ungünstige viszerale Fettgewebe reduziert. Mit Liraglutid plus Metformin nahm das viszerale Fett um $-17,1 \%$ bzw. $-16,4 \%$ ab im Vergleich $\mathrm{zu}-4,8 \%$ mit Sulfonylharnstoff plus Metformin (Jendle et al. Diab Obes Metabol 2009). „GLP-1-Analoga wirken sich also auf sämtliche kardiovaskuläre Risikofaktoren positiv aus “, so Marx.
Eine Frage ist aber noch offen: Kann auch die kardiovaskuläre Mortalität und Morbidität gesenkt werden? Die retrospektive Major Adverse Cardiovascular Events (MACE)-Analyse lässt das vermuten. Phase-II- und III-Studien mit 6638 Patienten wurden dafür gepoolt. 4257 erhielten Liraglutid. Die Studienautoren verglichen die Inzidenz kardiovaskulärer Ereignisse (kardiovaskulärer Tod, Herzinfarkt und zerebrovaskuläre Ereignisse) bei Liraglutid-Therapie mit der unter den Vergleichssubstanzen. „Egal welche statistische Methode verwendet wurde, das Ergebnis war mit Liraglutid im Trend allerdings nicht signifikant - immer besser als das mit der Vergleichssubstanz", sagte Marx. Die prospektive internationale LEADER $^{\mathrm{Tm}}$-Studie mit über 9000 Typ-2-Diabetikern soll nun klären, ob mit Liraglutid die Rate kardiovaskulärer Ereignisse tatsächlich sinkt. Die Ergebnisse werden für 2015 oder 2016 erwartet.

Veronika Schlimpert

Quelle: Pressekonferenz am 9.5.2013 beim

Diabeteskongress in Leipzig, Veranstalter: Novo Nordisk

\section{Neue Arznei erleichtert multifaktorielle Therapie}

\begin{abstract}
Bei der Antidiabetikaauswahl für ein patientenzentriertes Therapiekonzept ist außer der antihyperglykämischen Wirkung das Vermeiden von Hypoglykämien und Gewichtszunahme zentral wichtig.
\end{abstract}

Die Differenzialtherapie bei Diabetes fokussiert heute auf multiple Determinanten des kardiometabolischen Risikos. Antidiabetika, die wie der SGLT-2-Inhibitor Dapagliflozin einen Gewichtsanstieg verhindern oder das Körpergewicht sogar senken, ein niedriges Hypoglykämierisiko haben und einfach anzuwenden sind, bilden die Grundlage einer patientenzentrierten, individualisierten Therapie, sagte Prof. Jochen Seufert vom Uniklinikum Freiburg. Dapagliflozin
(Forxiga ${ }^{\circledast}$ ) reduziert die Rückresorption von Glukose in der Niere durch eine selektive SGLT2-Inhibition. Dadurch wird überschüssige Glukose insulinunabhängig mit dem Harn ausgeschieden, so Prof. Guntram Schernthaner aus Wien. Das Gliflozin senkt $\mathrm{HbA}_{1 \mathrm{c}}$, das Gewicht und den Blutdruck bei geringem Hypoglykämierisiko, betonte Schernthaner. Der SGLT-2-Hemmer wirke in jedem Stadium des Typ-2-Diabetes. In Kombination mit Metformin reduziert Dapagliflozin
$(10 \mathrm{mg} / \mathrm{d})$ den $\mathrm{HbA}_{1 \mathrm{c}}$ nach 24 Wochen um 0,84\%-Punkte im Vergleich zum Ausgangswert (vs.-0,30\%-Punkte bei Metformin plus Placebo). Die $\mathrm{HbA}_{1 \mathrm{c}}-\mathrm{Sen}$ kung war auch im Langzeitverlauf über zwei Jahre konstant. Das Gewicht sank im Vergleich zu Placebo signifikant um 2 $\mathrm{kg}$ nach 24 Wochen und um 3,1 kg nach 102 Wochen. Auch als Add-on-Therapie zu Insulin führte der SGLT-2-Hemmer zu einer über zwei Jahre anhaltenden $\mathrm{HbA}_{1 \mathrm{c}}{ }^{-}$ und Gewichtsabnahme. Zudem sank der Insulinbedarf.

Abdol A. Ameri

Quelle: Satellitensymposium beim Diabeteskongress am 8.5.2013 in Leipzig, Veranstalter: BristolMyers Squibb und AstraZeneca 